\title{
Emotional Data Collection using Self-Reporting Tools in Distance Learning Courses
}

\author{
Elise Lavoué \\ IAE Lyon, Université Jean Moulin Lyon 3 \\ LIRIS, CNRS5205, F-69355, France \\ Email: elise.lavoue@univ-lyon3.fr
}

\author{
Gaelle Molinari \\ UniDistance \\ 3960, Sierre, Suisse \\ Email: gaelle.molinari@unidistance.ch
}

\author{
Maxence Trannois \\ IAE Lyon, Université Jean Moulin Lyon 3 \\ LIRIS, CNRS5205, F-69355, France \\ Email: maxence.trannois@gmail.com
}

\begin{abstract}
Emotions have a strong impact on learning processes and regulation strategies. Increasing learners' awareness on their own emotions and group ones, has the potential to significantly improve learning processes. However, the collection of emotional data is challenging, especially in ecological contexts like distance learning and MOOCs. The lack of generic methods and tools for emotional data collection is a crucial issue for the research community. In this paper we present two self-reporting tools we have developed for distance learning. EMORE-L enables to collect emotions from learners through a focused daily questionnaire. CATE supports emotions sharing via emoticons associated to annotations in documents. We conducted two studies in the same Psychology curriculum to compare the type, the number and the co-occurence of emotions reported by learners with each tool. We discuss the differences we observed according to the tool and to the learning situation, so as to bring researchers and practitioners with a list of factors that may influence the emotional data collection process.
\end{abstract}

Index Terms-self-reporting tool, emotions, distance learning, data collection.

\section{INTRODUCTION}

Learners' emotions have a major impact on learning processes and regulation strategies [1]. Improving emotional awareness can help learners to regulate their own emotionsProviding learners with emotional awareness implies first to be able to collect data on their emotions. However, the collection of emotional data is challenging, especially in ecological contexts like distance learning and MOOCs. The lack of standard method for emotional data collection in such contexts is an issue for our community, if we build upon previous work, and to compare data across contexts. Most of data on learners' emotions is collected through laboratory experiments with measuring tools that can be not available in real situations.

Our work more addresses the design of self-reporting tools to collect data on emotions felt by learners in distance learning situations. Such tools should be designed to adapt to the characteristics of the learning situation and also to make the emotion awareness activity as easy as possible, the aim remaining to not interfere with the learning task. In this paper we present two self-reporting tools we have developed: EMORE-L and CATE. We report on two studies that have been conducted in a same distance learning curriculum within the Emoviz project [2]. The data analysis focuses on the following question for each study: what emotional data are collected on learner emotions?

\section{THEORETICAL BACKGROUND}

Our work is grounded in a cognitive approach to emotions, considered as the result of an appraisal process [3]. We find Pekruns work on emotions, their antecedents and effects in learning contexts, especially useful. He distinguishes between types of emotions [4]: (1) achievement emotions linked to the learning activity and its outcome (success or failure); (2) epistemic emotions related to the cognitive processing of the learning material, e.g. confusion experienced in a state of cognitive disequilibrium (3) emotions generated by the topic to learn; and (4) social emotions involved in the learners interaction with others as well as with their teachers.

Data collected on user emotions can be grouped into three categories [5]: perception-based estimations, physiological estimations and subjective feelings. We focus on self-report of emotions or subjective feelings, which is a less technologydependent method, and which can be more easily deployed remotely and on a large scale. Several types of self-reporting tools are proposed in the literature [5], using text-based questionnaires, visual instruments, colours or a combination of these means. Most tools have been developed on an ad-hoc basis, and are not adaptable or generalizable for other situations. Furthermore, it is difficult to determine the impact of a given tool and data collection process on the emotional data collected. For instance, using the Emotion Module proposed by Ruiz et al. [6], some learners may have been influenced by the colours, some others by the associated text and the others by the emoticons. As a consequence, the emotions reported by the learners may be of different nature, which may impair the integrity of the data collected. The collection of emotional data is challenging and the lack of method for emotional data collection in such contexts is a problem.

\section{ConteXt AND METhodology}

We conducted this research within the interdisciplinary project Emoviz involving researchers in Computer Science, Information Visualization and Psychology. The project aims to develop models, methods and tools to collect and visualize emotional information to support learners' regulation in distance learning. We focus this paper on the first stage of the project dedicated to methodologies and tools for self-reports of emotional data. 
We developed two emotion self-reporting tools: EMOREL (EMOtion REport for E-Learning) and CATE (Collaborative Annotation Tool for Emotions). We designed the tools to adapt to the characteristics of the learning situation and to not interfere with the learning task. Learners can select emotions among a list of 8 emotions recognized as specific to learning contexts [7]: delight, anxiety, curiosity, boredom, engagement, confusion, surprise and frustration. EMORE-L is a tool for a retrospective report of emotions on the activity and its results. The intensity of each emotion is reported using likert-scales. Emotions in this situation are rather achievement emotions. CATE is a tool for reporting emotions in real-time during reading using emoticons associated with annotations. Emotions here are rather emotions related to the content of the text (topic emotions) as well as to the cognitive states of learners (epistemic emotions).

We conducted two studies in the same distance learning curriculum in Psychology. The first study involved 15 students during 14 days. We asked them to use EMORE-L on a daily basis. The second study involved 22 students who used CATE during 13 days to annotate two documents given as resources for their course. The data analysis focuses on the type(s) of emotion based on the list of 8 emotions described above; the number of emotions expressed by the students in a same reporting item (questionnaire or annotation); the co-occurrence of emotions in same item.

\section{RESULTS AND DISCUSSION}

We observe that the type of emotions reported by the students are very different in the two studies, except for delight that was often expressed in both cases. Engagement was often reported in the second study, but it was very little expressed in the first one. Conversely, while anxiety was often expressed in the first study, it was not expressed much in the second study. More generally, learners mainly reported positive emotions with CATE, whereas they reported both negative and positive ones using EMORE-L. Regarding the cooccurence of emotions, results are also very different in both studies. While learners associated positive (delight, surprise) and negative (anxiety) emotions in the first study, they mainly associated positive emotions (engagement and curiosity) in the second study. Concerning the number of emotions, students reported several emotions ( 3 to 5) when using the EMORE-L likert scales with different associated intensity. In the CATE study, students were much more likely to associate each of their annotations with only one emotion using the emoticons.

We think these differences are mainly due to the procedure and more precisely to the learning situation in which students reported their emotions. First, learners may have been influenced by the fact that annotations are socially shared when using CATE. Sharing emotions may incite learners to express mostly positive emotions, due to social comparisons. This result was also observed in the study conducted in previous research [8]. Secondly, the frequency and the context of the data collection may have influenced the types of emotion expressed by learners. With EMORE-L, students reported their emotions once a day. Although the questions were often asked during a time slot dedicated to learning, we may have collected information closer to the daily mood than the discrete emotions felt at a precise moment. With CATE, the discrete emotions are more contextualized and depend on particular parts of the document. This can explain why students reported more emotions with EMORE-L than with CATE.

The timing of users reflecting on their emotions may also have an impact on the reports. Two kinds of reflections can be distinguished: reflection in action, i.e. emotions felt while conducting the activity, and reflection on action, i.e., emotions felt when thinking about previous activities [8]. With the experience sampling method used in the first study, students may have reported the emotions they felt during the day, and not the emotions they were currently feeling when completing the EMORE-L questionnaire. The emotional data collected in that case may be related to reflection on action. By using CATE, students reported the emotions they felt when annotating a given document. In that case, the emotional data is related to reflection in action.

Finally, we think that the types of emotions reported using the tools also depend on the focus of the emotions [4]. In the EMORE-L study, students were asked to report the emotions they experienced in respect to the learning activity, that is, their achievement emotions. In the CATE study, the focus of emotions was different as they could be triggered either by the topic (topic emotions) or by the cognitive processing of the documents (epistemic emotions).

\section{ACKNOWLEDGMENT}

This research has been funded within the Emoviz project by the Région Auvergne-Rhone-Alpes. The authors would like to thank the students who participated to the studies.

\section{REFERENCES}

[1] R. Pekrun, "The Control-Value Theory of Achievement Emotions: Assumptions, Corollaries, and Implications for Educational Research and Practice," Educational Psychology Review, vol. 18, no. 4, pp. 315-341, 2006.

[2] G. Molinari, M. Trannois, A. Tabard, and E. Lavoué, "Emore-1: an emotion reporting tool for distance learning," in Actes de la 28ième conférence francophone sur l'Interaction Homme-Machine. ACM, 2016, pp. 167-176.

[3] K. R. Scherer, A. Schorr, and T. Johnstone, Appraisal Processes in Emotion: Theory, Methods, Research. Oxford University Press, 2001.

[4] R. Pekrun and L. Linnenbrink-Garcia, "Academic Emotions and Student Engagement," in Handbook of Research on Student Engagement, S. L. Christenson, A. L. Reschly, and C. Wylie, Eds. Springer, 2012, pp. 259-282.

[5] D. Cernea and A. Kerren, "A survey of technologies on the rise for emotion-enhanced interaction," Journal of Visual Languages \& Computing, vol. 31, Part A, pp. 70-86, Dec. 2015.

[6] S. Ruiz, S. Charleer, M. Urretavizcaya, J. Klerkx, I. Fernndez-Castro, and E. Duval, "Supporting Learning by Considering Emotions: Tracking and Visualization a Case Study," in Proceedings of the Sixth International Conference on Learning Analytics \& Knowledge, ser. LAK 2016. New York, NY, USA: ACM, 2016, pp. 254-263.

[7] S. DMello, B. Lehman, R. Pekrun, and A. Graesser, "Confusion can be beneficial for learning,' Learning and Instruction, vol. 29, pp. 153-170, 2014.

[8] E. Lavoue, G. Molinari, Y. Prie, and S. Khezami, "Reflection-in-action markers for reflection-on-action in Computer-Supported Collaborative Learning settings," Computers \& Education, vol. 88, pp. 129-142, 2015. 